\title{
The Rise of Mitochondria in Peripheral Arterial Disease Physiopathology: Experimental and Clinical Data
}

\author{
Mégane Pizzimenti ${ }^{1,2}$, Marianne Riou ${ }^{1,2}$, Anne-Laure Charles ${ }^{1}$, Samy Talha 1,2 ${ }^{\mathbb{D}}$, \\ Alain Meyer 1,2, Emmanuel Andres ${ }^{3}\left(\mathbb{D}\right.$, Nabil Chakfé 1,4 , Anne Lejay ${ }^{1,4}$ and Bernard Geny ${ }^{1,2, *}$ \\ 1 Unistra, Translational Medicine Federation of Strasbourg (FMTS), Faculty of Medicine, Team 3072 \\ «Mitochondria, Oxidative Stress and Muscle Protection», 11 Rue Humann, 67000 Strasbourg, France; \\ megane.pizzimenti@hotmail.fr (M.P.); marianne.riou@chru-strasbourg.fr (M.R.); \\ anne.laure.charles@outlook.fr (A.-L.C.); samy.talha@chru-strasbourg.fr (S.T.); \\ alain.meyer1@chru-strasbourg.fr (A.M.); nabil.chakfe@chru-strasbourg.fr (N.C.); \\ anne.lejay@chru-strasbourg.fr (A.L.) \\ 2 Physiology and Functional Exploration Service, University Hospital of Strasbourg, 1 Place de l'Hôpital, \\ 67091 Strasbourg CEDEX, France \\ 3 Internal Medicine, Diabete and Metabolic Diseases Service, University Hospital of Strasbourg, \\ 1 Place de 1'Hôpital, 67091 Strasbourg CEDEX, France; emmanuel.andres@chru-strasbourg.fr \\ 4 Vascular Surgery and Kidney Transplantation Service, University Hospital of Strasbourg, \\ 1 Place de l'Hôpital, 67091 Strasbourg CEDEX, France \\ * Correspondence: bernard.geny@chru-strasbourg.fr
}

Received: 10 November 2019; Accepted: 29 November 2019; Published: 2 December 2019

\begin{abstract}
Peripheral arterial disease (PAD) is a frequent and serious condition, potentially lifethreatening and leading to lower-limb amputation. Its pathophysiology is generally related to ischemia-reperfusion cycles, secondary to reduction or interruption of the arterial blood flow followed by reperfusion episodes that are necessary but also-per se-deleterious. Skeletal muscles alterations significantly participate in PAD injuries, and interestingly, muscle mitochondrial dysfunctions have been demonstrated to be key events and to have a prognosis value. Decreased oxidative capacity due to mitochondrial respiratory chain impairment is associated with increased release of reactive oxygen species and reduction of calcium retention capacity leading thus to enhanced apoptosis. Therefore, targeting mitochondria might be a promising therapeutic approach in PAD.
\end{abstract}

Keywords: peripheral arterial disease; ischemia-reperfusion; mitochondria; oxidative stress; reactive oxygen species; antioxidant; calcium retention capacity; apoptosis

\section{Introduction}

Peripheral arterial diseases (PAD) is a major concern for public healthcare, affecting more than 200 million individual worldwide [1,2]. Its prevalence varies from $3 \%$ to $10 \%$, but can reach up to $20 \%$ in the elderly population [3].

PAD is defined by a narrowing of the peripheral arterial vasculature. It mostly affects lower limbs, leading to overall functional disability and reduced quality of life. Initially asymptomatic, PAD progressively compromises lower limb vascularization, leading to obstruction of the vessels by atheroma. PAD includes all stages of the disease, from asymptomatic with abolition of distal pulses, to intermittent claudication or critical limb threatening ischemia (CLTI) characterized by rest pain and/or ulcers. PAD is also often associated with cognitive dysfunction characterized by reduced performance in nonverbal reasoning, reduced verbal fluency, and decreased information 
processing speed [4]. Thus, PAD is a serious condition threatening both limb (risk of amputation) and vital prognosis of the patients. Indeed, despite recent therapeutic progress, morbidity and mortality rates remain incompressible around $20 \%$ and $15 \%$ five years after a diagnosis of symptomatic or asymptomatic PAD [5]. On average, the life expectancy of claudicating patients is reduced by 10 years, with a majority of death attributable to cardiovascular causes.

The treatment of PAD is mainly based on revascularization of the ischemic limb [6]. Nevertheless, this surgical procedure is not always possible, notably when the vascular state is too precarious or when the local evolution is too advanced. It appears therefore important to better understand PAD pathophysiology to offer optimal patient care. Indeed, insufficient oxygen supply was long presumed to be the main and sole cause for PAD symptoms. However, recent advances in understanding PAD physiopathology identified mitochondria as a key element in the deleterious process of PAD $[7,8]$.

Thus, skeletal muscles alterations significantly participate in PAD injuries, modulating its prognosis, and this review aims to describe muscle mitochondrial dysfunctions. Particularly, we will analyze data focused on mitochondrial respiratory chain respiration, on reactive oxygen species release and on mitochondrial calcium retention capacity which decrease is associated with enhanced apoptosis (Figure 1).

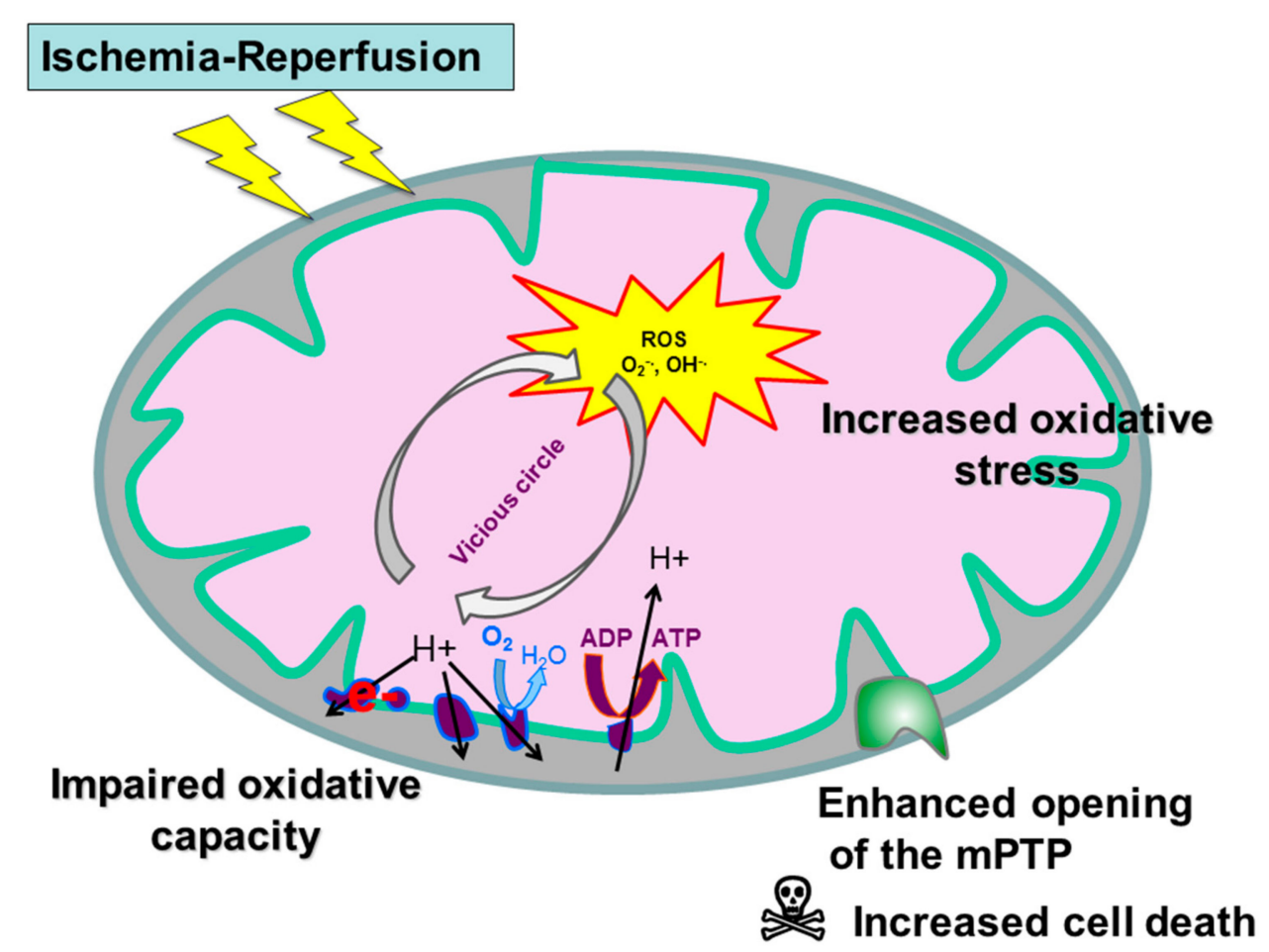

Figure 1. Mitochondrial dysfunction during peripheral arterial disease (PAD). MPTP: mitochondrial permeability transition pore.

\section{Mitochondrial Function under Normal and Pathological Conditions}

\subsection{Normal Condition}

Life requires energy, and this energy is stored in adenosine triphosphate (ATP) molecules, that are produced in the mitochondria by oxidative phosphorylation and assessed through mitochondrial respiration determination. Specifically, the oxidation of nutrients through the Krebs cycle provides reduced coenzymes (the reduced form of nicotinamide adenine dinucleotide (NADH)) and to a lesser extent flavin adenine dinucleotide $\left(\mathrm{FADH}_{2}\right)$, which are electron donors. This flow of electrons is supported by different redox reactions provided by the four complexes of the mitochondrial respiratory chain, up to the reduction of molecular oxygen in water. The respiratory complexes 
use the energy generated by this electron transfer to allow an active translocation of protons from the matrix to the inter-membrane mitochondrial space. This expulsion of protons results in the generation of a concentration gradient and a mitochondrial membrane potential across the inner membrane. ATP synthases use the transmembrane protonmotive force as a source of energy to drive a mechanical rotary mechanism leading to the chemical synthesis of ATP from ADP and Pi.The $\mathrm{F}_{1} \mathrm{~F}_{0}$ ATP synthase enzymes allow proton flux and ATP synthesis through a and $\mathrm{c}$ subunits of the $\mathrm{F}_{0}$ domain. The maintenance of this electrochemical gradient, also called protomotive force, is an essential element for the energetic role of the mitochondria [9-11].

Mitochondrial respiration generates free radicals derived from oxygen, the reactive oxygen species (ROS). A free radical is a chemical species containing an unpaired electron. Extremely unstable, this compound can react with more stable molecules to match its electron. It can then pull out an electron and behave as an oxidant, usually leading to the formation of new radicals in the chain and causing significant cell damage. Main ROS comprise superoxide anion, the hydroxyl radical and the highly reactive compound hydrogen peroxide $\left(\mathrm{H}_{2} \mathrm{O}_{2}\right)$. Detoxification systems exist, enzymatic (superoxide dismutase, catalase, glutathione peroxidase) or not (vitamins and trace elements).

Nevertheless, when the radical production remains contained below a certain threshold, the ROS activate defenses pathways involving the development of cellular antioxidants and mitochondrial biogenesis. Also named mitohormesis, these mechanisms constitute one of the therapeutic targets that can limit the lesions linked to repeated cycles of ischemia-reperfusion in PAD [12].

\subsection{Ischemic Condition}

During ischemia, ATP is generated by anaerobic glycolysis, leading to glycogen storage depletion, anaerobic metabolism activation and local lactic acidosis. The resulting depletion of ATP reduces the function of membrane pumps and causes cellular edema. Indeed, the cell tends to correct the acidosis by expelling the $\mathrm{H}+$ ions via the $\mathrm{Na}+\mathrm{H}+$ exchanger, thus saturating the cytoplasm with $\mathrm{Na}+$ ions and causing an osmotic shift to the cytoplasm. Cell edema is aggravated by $\mathrm{Na}+\mathrm{K}+\mathrm{ATP}$-dependent exchanger dysfunction due to lack of ATP, which also leads to Na+ accumulation in the cytoplasm. Acidosis also activates mediators, such as phospholipaseA2, that metabolize membrane phospholipids to arachidonic acid, a precursor of inflammatory mediators such as leukotrienes and prostaglandins. Ischemia will also initiate conversion of xanthine dehydrogenase to xanthine oxidase [13].

Reperfusion is able to prevent the irreversible damages of ischemia. Nevertheless, this process also generates lesions that aggravate the pre-existing tissue damages. At the cellular level, reoxygenation interrupts the lesions induced by ischemia, but causes reperfusion injury. During the first few minutes of reperfusion, the rapid correction of acidosis increases the cytosolic $\mathrm{Ca}^{2+}$, thus promoting the opening of the mitochondrial permeability transition pore [14]. This opening causes a sudden change in the mitochondrial membrane permeability, resulting in energy collapse incompatible with cell survival and inducing the release of pro-apoptotic factors from the inter-membrane mitochondrial space to the cytosol, leading to cell death. It is the intrinsic mitochondrial apoptosis pathway.

In parallel, reperfusion generates massive oxidative stress since xanthine oxidase and succinate, produced during ischemia, catalyzes the formation of uric acid from hypoxanthine and of ubiquinol, respectively, accompanied by the formation of large amounts of free radicals. Very interestingly, Chouchani et al. demonstrated a conserved metabolic response of tissues to ischemia-reperfusion (IR) revealing that reducing ischemia-induced succinate increase and its oxidation after reperfusion might be an important therapeutic during IR settings. [15-17].

The ROS thus produced exceed the cellular antioxidant defenses creating a vicious circle. Production of free radicals will cause a dysfunction of the mitochondrial respiratory chain, which in turn generate more ROS. Such ROS overproduction leads to several deleterious effects: lipid peroxidation, protein oxidation and DNA mutations, but also to the opening of the mitochondrial permeability transition pore. 


\section{Mitochondrial Oxidative Capacities in PAD}

\subsection{Experimental Data}

Impairments in mitochondrial respiration were observed in both claudicating and CLTI experimental models. Indeed, significant reduction in mitochondrial complexes I, II and IV activities was found in muscles of rats submitted to hindlimb ischemia-reperfusion compared to contralateral muscles [18-21]. Similarly, mouse models of CLTI presented decreased activities of the complexes I, III and IV in ischemic muscles compared with controls [22].

The respiratory impairments were shown to be strain-, muscle-, age- and disease- specific. Indeed, mitochondrial respiration was affected by hindlimb ischemia-reperfusion in limb muscles of BALB/C mice, but not of C57BL/6 mice [23]. Secondly, ischemia-reperfusion injury was shown to affect more severely the respiration in glycolytic muscles than in oxidative ones [24]. Furthermore, the impairments in mitochondrial respiration observed in young mice were greater in older animals submitted to ischemia-reperfusion injury [25]. Lastly, the decline in mitochondrial oxidative capacity was more severe in diabetic rats compared to non-diabetic animals (Table 1) [26].

Table 1. Mitochondrial oxidative capacity and peripheral arterial disease in selected experimental studies.

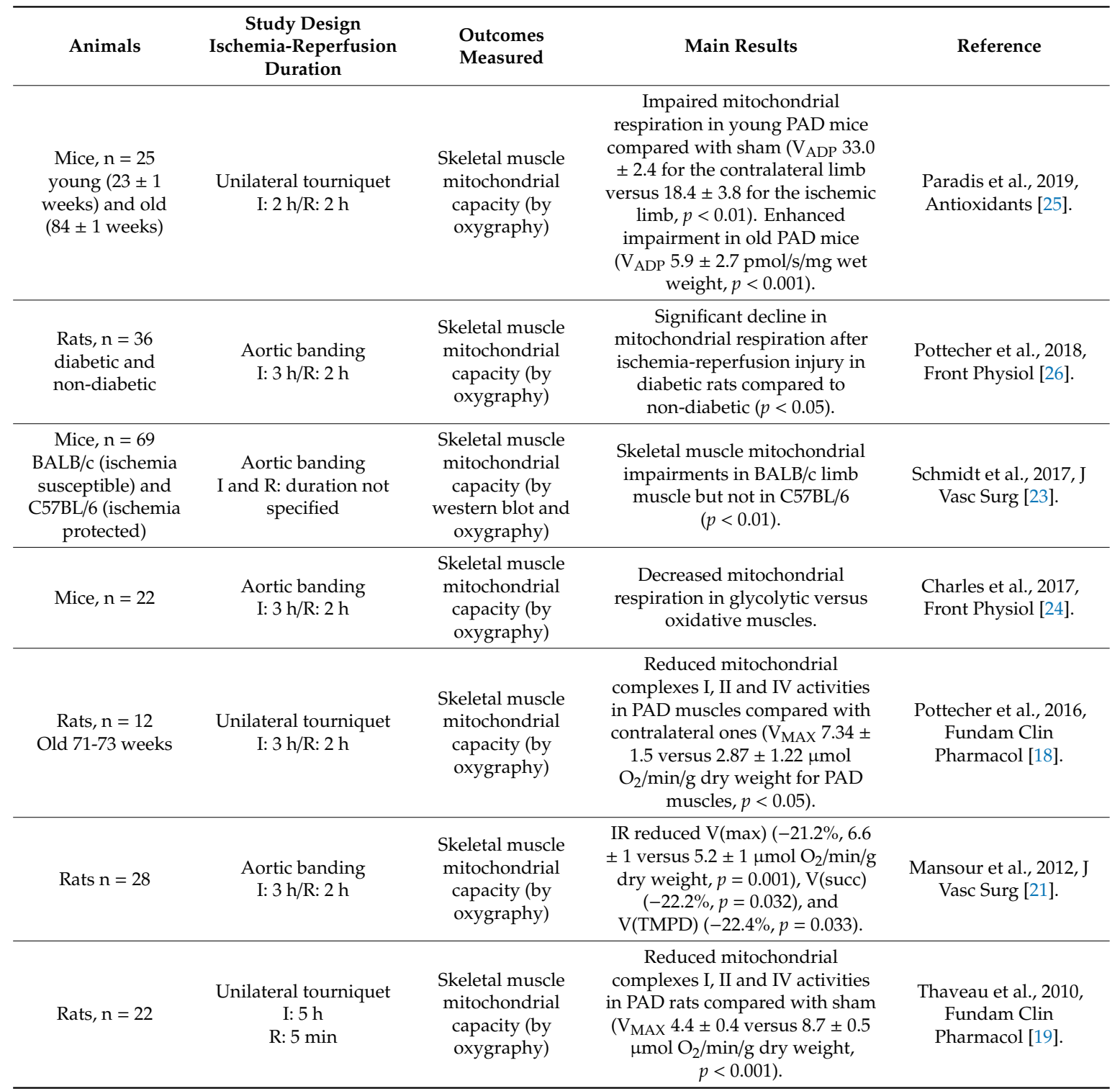


Table 1. Cont.

\begin{tabular}{|c|c|c|c|c|}
\hline Animals & $\begin{array}{c}\text { Study Design } \\
\text { Ischemia-Reperfusion } \\
\text { Duration }\end{array}$ & $\begin{array}{l}\text { Outcomes } \\
\text { Measured }\end{array}$ & Main Results & Reference \\
\hline Mice, $\mathrm{n}=48$ & $\begin{array}{c}\text { CLTI } \\
\text { Sequential left femoral } \\
\text { and iliac ligations } \\
\text { R: week } 12\end{array}$ & $\begin{array}{l}\text { Skeletal muscle } \\
\text { mitochondrial } \\
\text { capacity (by } \\
\text { oxygraphy) }\end{array}$ & $\begin{array}{l}\text { Reduced activity of complexes I } \\
\text { (by } 34 \% \text { ), III (by } 45 \% \text { ) and IV (by } \\
42 \% \text { ) in ischemic muscles } \\
\text { compared with controls } \\
(p<0.05) .\end{array}$ & $\begin{array}{c}\text { Pipinos et al., 2008, Am } \\
\text { J Physiol Regul Integr } \\
\text { Comp Physiol [22]. }\end{array}$ \\
\hline Rats, $n=20$ & $\begin{array}{l}\text { Unilateral tourniquet } \\
\text { I: } 5 \mathrm{~h}\end{array}$ & $\begin{array}{l}\text { Respiration of } \\
\text { isolated } \\
\text { mitochondria (by } \\
\text { polarographic } \\
\text { analysis) }\end{array}$ & $\begin{array}{l}\text { Inhibition of the mitochondrial } \\
\text { respiratory chain }\end{array}$ & $\begin{array}{l}\text { Brandão et al., J Surg } \\
\text { Res. } 2003 \text { [20]. }\end{array}$ \\
\hline
\end{tabular}

CLTI: critical limb threatening ischemia; I: ischemia; PAD: peripheral arterial disease; R: reperfusion. TMPD, N, N,

$\mathrm{N}^{\prime}, \mathrm{N}^{\prime}$-tetramethyl-p-phenylenediamine dihydrochloride.

\subsection{Clinical Data}

Oxygraphy measurements in PAD patients revealed significantly altered respiratory activity, notably of complexes I, III and IV, and of the acceptor control ratio [27-30]. Interestingly, more recent studies reported no difference in the mitochondrial respiration rate between PAD patients and healthy controls, despite alterations in $\mathrm{O}_{2}$ delivery, tissue-reoxygenation and ATP synthesis rate during exercise [31,32]. These conflicting findings could be explained by disparities in disease severity. Indeed, the alterations in mitochondrial oxidative capacity may have been the result of factors associated with higher morbidity in PAD, such as sarcopenia [33-35].

Mitochondrial energy metabolism was shown to decrease in PAD patients compared to controls [36, 37]. In contrast, Hou et al. found similar mitochondrial ATP production rate in both PAD patients and healthy controls [38]. Again, such differences might be explained by differences in disease severity or morbidity factors rate.

It is important to note that patients suffering from both PAD and type II diabetes (DT2) are more susceptible to reduced $\mathrm{O} 2$ consumption and mitochondrial oxidative phosphorylation compared to patients with PAD alone or to controls (Table 2) [39,40].

Table 2. Mitochondrial oxidative capacities and peripheral arterial disease in clinical studies.

\begin{tabular}{|c|c|c|c|c|}
\hline Population & $\begin{array}{c}\text { Number Studied } \\
\text { (Symptomatic/Controls) }\end{array}$ & Outcomes Measured & Main Results & Reference \\
\hline $\begin{array}{l}\text { Early stage } \\
\text { PAD }\end{array}$ & $10 / 11$ & $\begin{array}{l}\mathrm{O}_{2} \text { delivery, tissue } \\
\text { oxygenation and Vmax } \\
\text { (by high-resolution } \\
\text { respirometry). } \\
\text { Skeletal muscle } \\
\text { mitochondrial capacity } \\
\text { (by oxygraphy) }\end{array}$ & $\begin{array}{l}\text { PAD patients exhibited } \\
\text { significantly lower } \mathrm{O}_{2} \text { delivery } \\
(p<0.05) \text {, tissue-reoxygenation } \\
(58 \pm 3 \% \text { for controls versus } 44 \\
\pm 3 \% \text { for PAD patients, } p<0.05) \\
\text { and Vmax }(p<0.05) \text { during } \\
\text { exercise, compared with healthy } \\
\text { controls. } \\
\text { No differences were found in the } \\
\text { mitochondrial respiration rate. }\end{array}$ & $\begin{array}{c}\text { Hart et al., 2018, Am J } \\
\text { Physiol Heart Circ } \\
\text { Physiol [31]. }\end{array}$ \\
\hline $\begin{array}{l}\text { Claudicant } \\
\text { PAD }\end{array}$ & $10 / 12$ & $\begin{array}{l}\text { Skeletal muscle } \\
\text { mitochondrial capacity } \\
\text { (by oxygraphy) }\end{array}$ & $\begin{array}{l}\text { No differences were found in } \\
\text { the mitochondrial respiration } \\
\text { rate between PAD patients and } \\
\text { healthy controls. }\end{array}$ & $\begin{array}{l}\text { Hart et al., 2018, Exp } \\
\text { Physiol [32]. }\end{array}$ \\
\hline $\begin{array}{l}\text { Claudicant } \\
\text { PAD; } \\
\text { claudicant } \\
\text { PAD + DT2 }\end{array}$ & $\begin{array}{c}15(\mathrm{PAD}) / 15 \text { (PAD + } \\
\mathrm{DT} 2) / 10 \text { (controls) }\end{array}$ & $\begin{array}{l}\text { Skeletal muscle } \\
\text { mitochondrial capacity } \\
\text { (by oxygraphy) }\end{array}$ & $\begin{array}{l}\text { Significant reduction in oxygen } \\
\text { consumption in the PAD+DT2 } \\
\text { group compared with the PAD } \\
\text { group or the control group } \\
(p<0.05) \text {. No differences were } \\
\text { found in the mitochondrial } \\
\text { respiration rate between PAD } \\
\text { patients and healthy controls. }\end{array}$ & $\begin{array}{l}\text { Lindegaard et al., 2017, } \\
\text { Int Angiol [39]. }\end{array}$ \\
\hline
\end{tabular}


Table 2. Cont

\begin{tabular}{|c|c|c|c|c|}
\hline Population & $\begin{array}{c}\text { Number Studied } \\
\text { (Symptomatic/Controls) }\end{array}$ & Outcomes Measured & Main Results & Reference \\
\hline $\begin{array}{l}\text { Patients with } \\
\text { low ABI }\end{array}$ & $\begin{array}{c}82(\mathrm{ABI} \text { of } 0.90 \text { to } 1.10) / 281 \\
(\mathrm{ABI} \text { of } 1.11 \text { to } 1.40)\end{array}$ & $\begin{array}{l}\text { Phosphocreatine recovery } \\
\text { (by phosphorus-31 } \\
\text { magnetic resonance } \\
\text { spectroscopy) }\end{array}$ & $\begin{array}{l}\text { Significantly lower muscle } \\
\text { mitochondrial energy } \\
\text { production in patients with } \\
\text { lower ABI, compared with those } \\
\text { with higher ABI ( } 20.8 \mathrm{~ms}^{-1} \text { for } \\
\text { higher ABI versus } 19.3 \mathrm{~ms}^{-1} \text { for } \\
\text { lower ABI, } p=0.015) .\end{array}$ & $\begin{array}{l}\text { AlGhatrif et al., 2017, J } \\
\text { Am Heart Assoc [36]. }\end{array}$ \\
\hline $\begin{array}{l}\text { PAD (no stage } \\
\text { specified) }\end{array}$ & $30 / 30$ & $\begin{array}{l}\text { Skeletal muscle } \\
\text { mitochondrial capacity } \\
\text { (by oxygraphy) }\end{array}$ & $\begin{array}{c}\text { PAD subjects presented } \\
\text { significantly lower respiratory } \\
\text { activity compared with controls } \\
\qquad(p<0.05)\end{array}$ & $\begin{array}{l}\text { Koutakis et al., 2015, J } \\
\text { Histochem } \\
\text { Cytochem [27]. }\end{array}$ \\
\hline $\begin{array}{l}\text { Claudicant } \\
\text { PAD + } \\
\text { neuropathy + } \\
\text { DT2 }\end{array}$ & $7 / 14$ & $\begin{array}{l}\text { Phosphocreatine recovery } \\
\text { (by phosphorus- } 31 \\
\text { magnetic resonance } \\
\text { spectroscopy) }\end{array}$ & $\begin{array}{l}\text { Reduced mitochondrial } \\
\text { oxidative phosphorylation in } \\
\text { DT2 patients with lower } \\
\text { extremity complications } \\
\text { (neuropathy and PAD) } \\
(p<0.05) .\end{array}$ & $\begin{array}{c}\text { Tecilazich et al., 2013, J } \\
\text { Vasc Surg [40]. }\end{array}$ \\
\hline $\begin{array}{l}\text { Claudicant } \\
\text { PAD; CLI }\end{array}$ & $25 / 16$ & $\begin{array}{c}\text { Skeletal muscle } \\
\text { mitochondrial capacity } \\
\text { (by spectrophotometry) }\end{array}$ & $\begin{array}{l}\text { Decreased activity of complexes } \\
\text { I, III and IV in PAD muscle } \\
\text { compared to control }(p<0.05) \text {. }\end{array}$ & $\begin{array}{l}\text { Pipinos et al., 2006, } \\
\text { Free Radic Biol } \\
\text { Med [28]. }\end{array}$ \\
\hline $\begin{array}{l}\text { Claudicant } \\
\text { PAD; CLI }\end{array}$ & $9 / 9$ & $\begin{array}{l}\text { Skeletal muscle } \\
\text { mitochondrial capacity } \\
\text { (by oxygraphy) }\end{array}$ & $\begin{array}{l}\text { Significantly lower respiratory } \\
\text { rates, and lower acceptor control } \\
\text { ratio }(2.90 \pm 0.20 \text { for controls } \\
\text { versus } 1.41 \pm 0.10 \text { for PAD) in } \\
\text { patients with PAD compared } \\
\text { with controls }(p<0.05) .\end{array}$ & $\begin{array}{l}\text { Pipinos et al., 2003, J } \\
\text { Vasc Surg [29]. }\end{array}$ \\
\hline $\begin{array}{l}\text { Claudicant } \\
\text { PAD }\end{array}$ & $7 / 11$ & $\begin{array}{l}\text { ATP synthesis (by } \\
\text { luminometer) }\end{array}$ & $\begin{array}{l}\text { Similar mitochondrial ATP } \\
\text { production rate were in PAD } \\
\text { patients and healthy controls. }\end{array}$ & $\begin{array}{l}\text { Hou et al., 2002, Clin } \\
\text { Physiol Funct } \\
\text { Imaging [38]. }\end{array}$ \\
\hline $\begin{array}{l}\text { Claudicant } \\
\text { PAD }\end{array}$ & $12 / 14$ & $\begin{array}{l}\text { Phosphocreatine and } \\
\text { ADP recovery (by } \\
\text { phosphorus-31 magnetic } \\
\text { resonance spectroscopy) }\end{array}$ & $\begin{array}{l}\text { Defective phosphocreatine ( } 44 \pm \\
3 \text { s for controls versus } 137 \pm 41 \mathrm{~s} \\
\text { for PAD) and ADP recovery } \\
\text { (29 } \pm 2 \text { sersus } 60 \pm 10 \text { s for } \\
\text { PAD) in PAD compared with } \\
\text { controls }(p<0.05) \text {. }\end{array}$ & $\begin{array}{l}\text { Pipinos et al., 2000, J } \\
\text { Vasc Surg [37]. }\end{array}$ \\
\hline
\end{tabular}

ABI: ankle brachial index; CLTI: critical limb threatening ischemia; DT2: type II diabetes; PAD: peripheral arterial disease.

\section{Reactive Oxygen Species Production, Proteins, Lipids and DNA Alterations and Impaired Antioxidant Defense, in PAD}

The interaction between mitochondria and oxidative stress in skeletal muscle is modulated by repeated cycles of ischemia-reperfusion in the context of PAD. The vascular damages create an imbalance between oxygen supply and demand during efforts, generating a situation of ischemia; followed by a situation of reperfusion when the patient is at rest. Repetition of ischemia and reperfusion cycles are deleterious for skeletal muscle and lead to myopathy and to remote organ damage $[7,28,41]$.

\subsection{Experimental Data}

ROS production was found increased in both animal models of PAD (acute ischemia-reperfusion and CLTI) as compared to controls, using either measurements of 1) free radical species by electron paramagnetic resonance spectroscopy, 2) dihydroethidium (DHE) by epifluorescence microscopy or 3) $\mathrm{H}_{2} \mathrm{O}_{2}$ by Amplex Red perioxide assay [24,42,43]. Interestingly, ROS production was greater in PAD animals presenting with hypercholesterolemia or diabetes [26,44]. These evidences suggest an association between PAD comorbidity factors and enhanced mitochondrial dysfunction. 
Furthermore, deleterious effects of oxidative stress were also observed in ischemic skeletal muscles, as highlighted by higher levels of oxidative stress markers (superoxide dismutase [45], protein carbonyls and 4-hydroxy-2-nonenanal protein (HNE) adducts) [22], and elevated DNA alterations [46].

Finally, antioxidant defenses have been shown to be impaired by ischemia-reperfusion. Indeed, alterations in the expression of superoxide dismutase 1 and 2 (SOD1 and SOD2), catalase and manganese superoxide dismutase (MnSOD) were observed in ischemic muscles compared with controls (Table 3) [22,45,47].

Table 3. Reactive oxygen species production during peripheral arterial disease in experimental studies.

\begin{tabular}{|c|c|c|c|c|}
\hline Animals & $\begin{array}{c}\text { Study Design } \\
\text { Ischemia-Reperfusion } \\
\text { Duration }\end{array}$ & Outcomes Measured & Main Results & Reference \\
\hline $\begin{array}{c}\text { Mice, } \mathrm{n}=7 \\
\text { ApoE-/- versus } \\
\text { ApoE }+/+\end{array}$ & $\begin{array}{c}\text { CLTI } \\
\text { Sequential right femoral } \\
\text { and iliac ligations. R: } \\
\text { day } 40\end{array}$ & $\begin{array}{c}\text { Free radical } \\
\text { measurement (by } \\
\text { electron paramagnetic } \\
\text { resonance spectroscopy) }\end{array}$ & $\begin{array}{c}\text { Enhanced ROS production in muscles } \\
\text { of ApoE-/- }(+63.6 \%) \text { and ApoE }+/+ \\
(+41.4 \%) \text { mice compared with } \\
\text { contralateral muscles. }\end{array}$ & $\begin{array}{l}\text { Lejay et al., 2019, } \\
\text { Eur J Vasc Endovasc } \\
\text { Surg [44]. }\end{array}$ \\
\hline $\begin{array}{c}\text { Rats, } \mathrm{n}=36 \\
\text { diabetic versus } \\
\text { non-diabetic }\end{array}$ & $\begin{array}{l}\text { Aortic banding } \\
\text { I: } 3 \mathrm{~h} / \mathrm{R}: 2 \mathrm{~h}\end{array}$ & $\begin{array}{l}\text { DHE measures of ROS } \\
\text { (by epifluorescence } \\
\text { microscopy) }\end{array}$ & $\begin{array}{l}\text { Increase in normalized DHE } \\
\text { fluorescence in diabetic PAD } \\
\text { compared to diabetic controls } \\
\qquad(p<0.001)\end{array}$ & $\begin{array}{l}\text { Pottecher et al., 2018, } \\
\text { Front Physiol [26]. }\end{array}$ \\
\hline Mice, $n=20$ & $\begin{array}{c}\text { CLTI } \\
\text { Left femoral ligation. } \\
\text { R: day } 21\end{array}$ & $\begin{array}{l}\text { mtDNA damage } \\
\text { quantification (by } \\
\text { quantitative PCR) }\end{array}$ & $\begin{array}{l}\text { Increase in mtDNA damages in } \\
\text { ischemic muscles of PAD mice } \\
\text { compared with sham }(p<0.05) \text {. }\end{array}$ & $\begin{array}{c}\text { Miura et al., 2017, Int J } \\
\text { Mol Sci [46]. }\end{array}$ \\
\hline Mice, $\mathrm{n}=20$ & $\begin{array}{l}\text { CLTI } \\
\text { Sequential right femoral } \\
\text { and iliac ligations. R: } \\
\text { day } 30\end{array}$ & $\begin{array}{l}\text { Antioxidant } \\
\text { quantification (by } \\
\text { quantitative PCR) }\end{array}$ & $\begin{array}{c}\text { Significant decrease in mRNA } \\
\text { expression of the antioxidant } \\
\text { enzymes SOD1 }(0.39 \pm 0.10 \text { for sham } \\
\text { limb versus } 0.10 \pm 0.06 \text { for ischemic } \\
\text { limb), SOD2 }(0.32 \pm 0.16 \text { versus } 0.11 \pm \\
0.07) \text { and catalase }(0.38 \pm 0.04 \text { versus } \\
0.22 \pm 0.11) \text { in ischemic muscles } \\
\text { compared with control ones }(p<0.05) \text {. }\end{array}$ & $\begin{array}{c}\text { Lejay et al., 2017, Front } \\
\text { Physiol [47]. }\end{array}$ \\
\hline Mice, $n=22$ & $\begin{array}{l}\text { Aortic banding } \\
\text { I: } 3 \mathrm{~h} / \mathrm{R}: 2 \mathrm{~h}\end{array}$ & $\begin{array}{c}\text { Free radical } \\
\text { measurement (by } \\
\text { electron paramagnetic } \\
\text { resonance spectroscopy) }\end{array}$ & $\begin{array}{l}\text { Ischemia-reperfusion injury increased } \\
\text { ROS production in ischemic muscles } \\
\text { compared to no ischemic contralateral } \\
(+79.15 \pm 28.72 \%, p=0.04)\end{array}$ & $\begin{array}{l}\text { Charles et al., 2017, } \\
\text { Front Physiol [24]. }\end{array}$ \\
\hline Mice, $n=6$ & $\begin{array}{c}\text { CLTI } \\
\text { Right femoral ligation. } \\
\text { R: day } 10\end{array}$ & $\begin{array}{c}\mathrm{H}_{2} \mathrm{O}_{2} \text { measurement (by } \\
\text { Amplex Red assay) }\end{array}$ & $\begin{array}{l}\text { Significant increase in } \mathrm{H}_{2} \mathrm{O}_{2} \text { level in } \\
\text { ischemic muscles compared with } \\
\text { sham ones }(p<0.05)\end{array}$ & $\begin{array}{c}\text { Kwon et al., 2016, Int J } \\
\text { Pharm [43]. }\end{array}$ \\
\hline Mice, $n=28$ & $\begin{array}{l}\text { CLTI } \\
\text { Sequential right femoral } \\
\text { and iliac ligations. R: } \\
\text { day } 30 .\end{array}$ & $\begin{array}{c}\text { Free radical } \\
\text { measurement (by } \\
\text { electron paramagnetic } \\
\text { resonance spectroscopy) } \\
\text { DHE measures of ROS } \\
\text { (by epifluorescence } \\
\text { microscopy) }\end{array}$ & $\begin{array}{l}\text { CLI induced a significant increase in } \\
\text { ROS production in ischemic muscles } \\
\text { compared with controls. DHE } \\
\text { staining was higher in ischemic } \\
\text { muscles }(p<0.01) .\end{array}$ & $\begin{array}{l}\text { Lejay et al., 2015, } \\
\text { Eur J Vasc Endovasc } \\
\text { Surg [42]. }\end{array}$ \\
\hline Rats, $\mathrm{n}=35$ & $\begin{array}{c}\text { Aortic banding } \\
\mathrm{I}: 2 \mathrm{~h} / \mathrm{R}: 10 \mathrm{~min} \text { and } 2 \mathrm{~h}\end{array}$ & $\begin{array}{l}\text { DHE staining (by } \\
\text { epifluorescence } \\
\text { microscopy) }\end{array}$ & $\begin{array}{l}\text { ROS increased significantly after } \\
\text { ischemia alone }(+324 \pm 66 \%, p= \\
0.038), \text { normalized after } 10 \text { min of } \\
\text { reperfusion, and increased again at } 2 \\
\text { h of reperfusion }(+349.2 \pm 67 \%, p= \\
\text { 0.024). Oxidative stress preceded } \\
\text { skeletal muscle mitochondrial } \\
\text { dysfunction. }\end{array}$ & $\begin{array}{l}\text { Guillot et al., 2014, J } \\
\text { Vasc Surg [48]. }\end{array}$ \\
\hline Mice, $n=18$ & $\begin{array}{l}\text { Unilateral tourniquet } \\
\text { I: } 3 \mathrm{~h} / \mathrm{R}: 4 \mathrm{~h}\end{array}$ & $\begin{array}{l}\text { Superoxide anion } \\
\text { production measurement } \\
\text { (by luminometer); } \\
\text { Quantification of } \\
\text { MnSOD (by Western } \\
\text { blot) }\end{array}$ & $\begin{array}{l}\text { Increased superoxide production and } \\
\text { decreased activity of the } \\
\text { mitochondria-targeted SOD isoform) } \\
\text { in the ischemia-reperfusion group. }\end{array}$ & $\begin{array}{l}\text { Tran et al., 2011, Eur J } \\
\text { Pharmacol [45]. }\end{array}$ \\
\hline Mice, $n=48$ & $\begin{array}{c}\text { CLTI } \\
\text { Sequential left femoral } \\
\text { and iliac ligations } \\
\text { R: week } 12\end{array}$ & $\begin{array}{l}\text { Protein carbonyls, HNE } \\
\text { adducts and MnSOD } \\
\text { expression quantification } \\
\text { (by reverse phase protein } \\
\text { lysate microarray) }\end{array}$ & $\begin{array}{l}\text { Significantly higher expression of } \\
\text { protein carbonyls, HNE adducts and } \\
\text { MnSOD in ischemic muscles } \\
\text { compared with controls }(p<0.05) \text {. }\end{array}$ & $\begin{array}{l}\text { Pipinos et al., 2008, Am } \\
\text { J Physiol Regul Integr } \\
\text { Comp Physiol [22]. }\end{array}$ \\
\hline
\end{tabular}

CLTI: critical limb threatening ischemia; DHE: dihydroethidium; dw: dry weight; HNE: 4-hydroxy-2-nonenal; I: ischemia; MnSOD: manganese superoxide dismutase; mtDNA: mitonchondrial DNA; PAD: peripheral arterial disease; PCR: polymerase chain reaction; R: reperfusion; ROS: reactive oxygen species; SOD: superoxide dismutase. 


\subsection{Clinical Data}

Similar to the findings on experimental models, PAD patients displayed increased mitochondria-derived ROS production characterized by elevated levels of free radical species [32].

Moreover, oxidative damages were also observed in patients suffering from PAD, as reported by higher levels of oxidative stress markers (protein carbonyl groups, HNE-protein adducts and lipid hydroperoxides), and elevated DNA alterations [28,49-53].

Lastly, evidence of reduced antioxidant defenses has been shown in PAD patients, notably with altered activities of the antioxidant enzymes SOD, catalase and glutathione peroxidase (Table 4) [28].

Table 4. Reactive oxygen species production during peripheral arterial disease in clinical studies.

\begin{tabular}{|c|c|c|c|c|}
\hline Population & $\begin{array}{c}\text { Number Studied } \\
\text { (Symptomatic/Controls) }\end{array}$ & Outcomes Measured & Main Results & Reference \\
\hline Claudicant PAD & $10 / 34$ & $\begin{array}{c}\text { Mitochondrial DNA copy } \\
\text { number (by quantitative } \\
\text { PCR) }\end{array}$ & $\begin{array}{l}\text { Significant association between } \\
\text { disease severity and increased } \\
\text { mitochondrial DNA copy } \\
\text { number }(p<0.05)\end{array}$ & $\begin{array}{c}\text { McDermott et al., 2018, } \\
\text { Vasc Med [49]. }\end{array}$ \\
\hline Claudicant PAD & $10 / 12$ & $\begin{array}{l}\text { Free radical measurement } \\
\text { (by electron paramagnetic } \\
\text { resonance spectroscopy) }\end{array}$ & $\begin{array}{c}\text { Significant increase in } \\
\text { mitochondria-derived ROS } \\
\text { production in PAD }(1.0 \pm 0.36 \\
\text { AU/mg tissue for controls versus } \\
4.3 \pm 1.0 \mathrm{AU} / \mathrm{mg} \text { tissue for PAD, } \\
p<0.05) .\end{array}$ & $\begin{array}{l}\text { Hart et al., 2018, Exp } \\
\text { Physiol [32]. }\end{array}$ \\
\hline $\begin{array}{l}\text { Claudicant PAD; } \\
\text { CLTI }\end{array}$ & $\begin{array}{l}28 \text { claudicants } / 25 \\
\text { CLTI/ } 25 \text { controls }\end{array}$ & $\begin{array}{l}\text { Carbonyl groups } \\
\text { quantification (by } \\
\text { quantitative fluorescence } \\
\text { microscopy) }\end{array}$ & $\begin{array}{l}\text { Observation of a } 25 \% \text { increase in } \\
\text { carbonyl groups (markers of } \\
\text { oxidative damage) in myofibers } \\
\text { of all PAD patients compared } \\
\text { with controls }(p<0.05)\end{array}$ & $\begin{array}{l}\text { Koutakis et al., 2014, } \\
\text { Redox Biol [50]. }\end{array}$ \\
\hline $\begin{array}{l}\text { Claudicant PAD; } \\
\text { CLTI }\end{array}$ & $34 / 21$ & $\begin{array}{c}\text { Carbonyl groups and HNE } \\
\text { adducts quantification (by } \\
\text { quantitative fluorescence } \\
\text { microscopy) }\end{array}$ & $\begin{array}{c}\text { Significant increase in carbonyl } \\
\text { groups }(30 \%, p<0.0001) \text { and } \\
\text { HNE adducts }(40 \%, p<0.0001) \\
\text { in PAD myofibers compared to } \\
\text { controls. }\end{array}$ & $\begin{array}{l}\text { Weiss et al., 2013, J } \\
\text { Transl Med [51]. }\end{array}$ \\
\hline $\begin{array}{l}\text { Claudicant PAD; } \\
\text { CLTI }\end{array}$ & $16 / 10$ & $\begin{array}{l}\text { Lipid hydroperoxides } \\
\text { measurement (by ferrous } \\
\text { oxidation/xylenol orange } \\
\text { technique); Protein } \\
\text { carbonyls measurments } \\
\text { (using an Enzyme } \\
\text { Immuno-Assay kit); } \\
\text { HNE detection } \\
\text { (by western blot); } \\
\text { Antioxidant activity (by } \\
\text { spectrophotometry) }\end{array}$ & $\begin{array}{c}\text { Higher levels of lipid } \\
\text { hydroperoxides }(12.45 \pm 0.74 \\
\text { mmol/g wet weight for controls } \\
\text { versus } 20.32 \pm 1.02 \text { for PAD), } \\
\text { protein carbonyls }(0.22 \pm 0.02 \\
\text { nmol/mg for controls versus } 0.35 \\
\pm 0.04) \text { and HNE }(191.2 \pm 7.17 \\
\text { total binding versus } 226.4 \pm \\
\text { 10.4) was found in PAD patients } \\
\text { compared to controls }(p<0.05) \text {. } \\
\text { Significant decrease in SOD } \\
\text { activity, and increase in catalase } \\
\text { and glutathione peroxidase } \\
\text { activities. }\end{array}$ & $\begin{array}{l}\text { Pipinos et al., } \\
\text { 2006, Free Radic Biol } \\
\text { Med [28]. }\end{array}$ \\
\hline Claudicant PAD & 9 claudicants & $\begin{array}{l}\text { Quantification of } \\
\text { mitochondrial DNA injury } \\
\text { (by PCR) }\end{array}$ & $\begin{array}{l}\text { Substantial injury to } \\
\text { mitochondrial DNA in PAD } \\
\text { patients occurring bilaterally in } \\
\text { patients with unilateral PAD. }\end{array}$ & $\begin{array}{c}\text { Brass et al., 2000, Vasc } \\
\text { Med [52]. }\end{array}$ \\
\hline Claudicant PAD & $8 / 10$ & $\begin{array}{l}\text { Quantification of } \\
\text { mitochondrial DNA injury } \\
\text { (by PCR) }\end{array}$ & $\begin{array}{c}\text { Accumulation of } 4977-\mathrm{bp} \\
\text { mitochondrial deletion } \\
\text { frequency in patients with PAD } \\
\text { compared with controls }(0.05 \pm \\
0.01 \% \text { for controls versus } 0.43 \pm \\
0.28 \% \text { for the less-affected limb } \\
\text { versus } 0.88 \pm 0.53 \% \text { for the } \\
\text { worse-affected limb, } p<0.05) \text {. }\end{array}$ & $\begin{array}{l}\text { Bhat et al., 1999, } \\
\text { Circulation [53]. }\end{array}$ \\
\hline
\end{tabular}

CLTI: critical limb threatening ischemia; HNE: 4-hydroxy-2-nonenal; mtDNA: mitochondrial DNA; PAD: peripheral arterial disease; PCR: polymerase chain reaction; SOD: superoxide dismutase.

\section{Mitochondrial Implication in Apoptosis during PAD}

\subsection{Experimental Data}

Rodent models of PAD displayed elevated protein expression of the apoptotic factors cleaved-caspase 3, cleaved-poly (ADP-robose) polymerase (PARD) and mitochondrial and cytosolic $\mathrm{Bcl}$-associated $\mathrm{X}(\mathrm{Bax})$, and reduced protein expression of the anti-apoptotic factor Bcl-2, compared 
with controls $[21,54,55]$. Furthermore, a decrease in mitochondrial calcium retention capacity was observed in ischemic limbs compared with contralateral ones (Table 5) $[25,44,47,56,57]$.

Table 5. Mitochondrial implication in apoptosis during peripheral arterial disease in experimental studies.

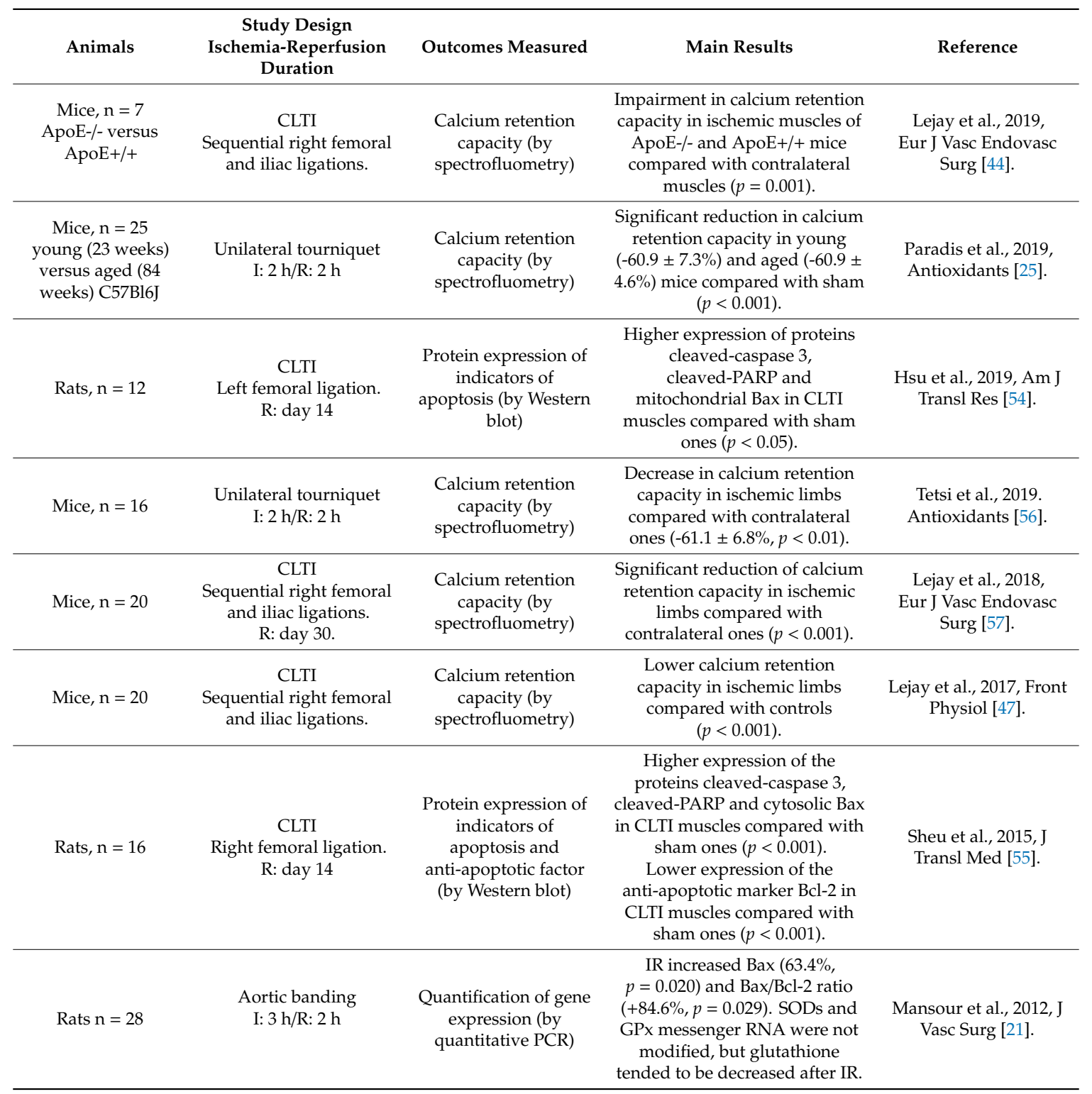

CLTI: critical limb threatening ischemia; I: ischemia; IR: ischemia-reperfusion; PAD: peripheral arterial disease; R: reperfusion.

\subsection{Clinical Data}

Human investigations also showed a clear implication of apoptosis in PAD pathophysiology. Indeed, PAD patients displayed elevated levels of genes mediating apoptosis [58], increased DNA fragmentation and caspase-3 activity [59]. Additionally, multiple studies reported higher levels of apoptotic cells in different cell types, notably endothelial cells and lymphocytes [60,61]. Interestingly, another study reported similar levels of endothelial apoptosis in both PAD and control groups. This result is likely due to disparities in patient's selection, with patients presenting with lower associated risk factors (Table 6) [62]. 
Table 6. Mitochondrial implication in apoptosis during peripheral arterial disease in clinical studies.

\begin{tabular}{|c|c|c|c|c|}
\hline Population & $\begin{array}{c}\text { Number Studied } \\
\text { (Symptomatic/Controls) }\end{array}$ & Outcomes Measured & Main Results & Reference \\
\hline Claudicant PAD & $130 / 36$ & $\begin{array}{c}\text { Caspase activity } \\
\text { measurement } \\
\text { (by caspase Assay) }\end{array}$ & $\begin{array}{l}\text { No difference observed in } \\
\text { apoptosis between the PAD and the } \\
\text { control group }(p=0.463) .\end{array}$ & $\begin{array}{l}\text { Gardner et al., 2014, } \\
\text { Angiology [62]. }\end{array}$ \\
\hline Claudicant PAD & $156 / 16$ & $\begin{array}{l}\text { Caspase activity } \\
\text { measurement } \\
\text { (by caspase Assay) }\end{array}$ & $\begin{array}{l}\text { Higher percentage of endothelial } \\
\text { cell apoptosis in the PAD group } \\
\text { compared with the control group } \\
(+164 \%, p<0.001)\end{array}$ & $\begin{array}{l}\text { Gardner et al., 2014, Int } \\
\text { J Vasc Med [60]. }\end{array}$ \\
\hline Claudicant PAD & $19 / 18$ & $\begin{array}{l}\text { Quantification of gene } \\
\text { expression (by } \\
\text { quantitative PCR) }\end{array}$ & $\begin{array}{c}\text { Upregulation in genes mediating } \\
\text { apoptosis: } B C L-2, G 0 S 2, K L F 6, \\
P T P 4 A 1 \text { and } C F L A R \text {. }\end{array}$ & $\begin{array}{l}\text { Masud et al., 2012, J } \\
\text { Clin Bioinforma [58]. }\end{array}$ \\
\hline Claudicant PAD & $10 / 10$ & $\begin{array}{l}\text { Detection and } \\
\text { quantification of } \\
\text { apoptosis (by } \\
\text { fluorescence microscopy) }\end{array}$ & $\begin{array}{l}\text { Higher percentage of late apoptotic } \\
\text { lymphocytes (by } 33 \% \text { ) in the PAD } \\
\text { patients compared with healthy } \\
\text { controls. }\end{array}$ & $\begin{array}{l}\text { Skórkowska-Telichowska } \\
\text { et al., 2009, Clin Invest } \\
\text { Med [61]. }\end{array}$ \\
\hline
\end{tabular}

PAD: peripheral arterial disease; PCR: polymerase chain reaction.

\section{Conclusions}

In summary, PAD is a public health issue even when poorly symptomatic [63], and mitochondria are importantly involved in its pathophysiology. Not only because mitochondrial alterations reduce the energy available for cell function, but also because mitochondria participate in the increased ROS production (and therefore to a greater oxidative stress) and in the enhanced opening of the mitochondrial permeability pore which favor the intrinsic pathway of cell apoptosis. These data account for the fact that skeletal muscle mitochondrial function might be considered as a prognostic factor in the setting of PAD in humans. On the other hand, if ROS production remains contained below a certain threshold, mitohormesis and stimulation of the antioxidant defenses can be protective supporting that mitochondrial function modulation might be a therapeutic target. Thus, further studies focused on mitochondria are warranted to optimize the care of patients presenting with PAD.

Author Contributions: Conceptualization, M.R., A.-L.C., A.L., and B.G.; methodology, M.P., M.R., A.-L.C., A.L., and B.G.; validation, M.P., M.R., A.-L.C., S.T., A.M., E.A., N.C., A.L., and B.G.; writing-original draft preparation, M.P., A.L., and B.G. writing-review and editing, M.P., N.C. A.L. and B.G.; supervision, A.L. and B.G.

Funding: This research received no external funding.

Conflicts of Interest: The authors declare no conflict of interest.

\section{References}

1. Criqui, M.H.; Aboyans, V. Epidemiology of peripheral artery disease. Circ. Res. 2015, 116, 1509-1526. [CrossRef]

2. Duff, S.; Mafilios, M.S.; Bhounsule, P.; Hasegawa, J.T. The burden of critical limb ischemia: A review of recent literature. Vasc. Health. Risk. Manag. 2019, 15, 187-208. [CrossRef] [PubMed]

3. Dua, A.; Lee, C.J. Epidemiology of Peripheral Arterial Disease and Critical Limb Ischemia. Tech. Vasc. Interv. Radiol. 2016, 19, 91-95. [CrossRef] [PubMed]

4. Leardini-Tristao, M.; Charles, A.L.; Lejay, A.; Pizzimenti, M.; Meyer, A.; Estato, V.; Tibiriçá, E.; Andres, E.; Geny, B. Beneficial Effect of Exercise on Cognitive Function during Peripheral Arterial Disease: Potential Involvement of Myokines and Microglial Anti-Inflammatory Phenotype Enhancement. J. Clin. Med. 2019, 8, 653. [CrossRef] [PubMed] 
5. Aboyans, V.; Ricco, J.B.; Bartelink, M.L.E.L.; Björck, M.; Brodmann, M.; Cohnert, T.; Collet, J.P.; Czerny, M.; De Carlo, M.; Debus, S.; et al. Editor's Choice-2017 ESC Guidelines on the Diagnosis and Treatment of Peripheral Arterial Diseases, in collaboration with the European Society for Vascular Surgery (ESVS). Eur. J. Vasc. Endovasc. Surg. 2018, 55, 305-368. [CrossRef]

6. Conte, M.S.; Bradbury, A.W.; Kolh, P.; White, J.V.; Dick, F.; Fitridge, R.; Mills, J.L.; Ricco, J.B.; Suresh, K.R.; Murad, M.H.; et al. Global Vascular Guidelines on the Management of Chronic Limb-Threatening Ischemia. Eur. J. Vasc. Endovasc. Surg. 2019, 58, S1-S109.e33. [CrossRef]

7. Paradis, S.; Charles, A.L.; Meyer, A.; Lejay, A.; Scholey, J.W.; Chakfé, N.; Zoll, J.; Geny, B. Chronology of mitochondrial and cellular events during skeletal muscle ischemia-reperfusion. Am. J. Physiol.-Cell Physiol. 2016, 310, C968-C982. [CrossRef]

8. Koutakis, P.; Ismaeel, A.; Farmer, P.; Purcell, S.; Smith, R.S.; Eidson, J.L.; Bohannon, W.T. Oxidative stress and antioxidant treatment in patients with peripheral artery disease. Physiol. Rep. 2018, 6, e13650. [CrossRef]

9. Walker, J.E. ATP Synthesis by Rotary Catalysis (Nobel lecture). Angew. Chem. Int. Edit. 1998, 37, $2308-2319$. [CrossRef]

10. Walker, J.E.; Dickson, V.K. The peripheral stalk of the mitochondrial ATP synthase. Biochim. Biophys. Acta (BBA)—Bioenerg. 2006, 1757, 286-296. [CrossRef]

11. Walker, J.E. The ATP synthase: The understood, the uncertain and the unknown. Biochem. Soc. Trans. 2013, 41, 1-16. [CrossRef] [PubMed]

12. Lejay, A.; Meyer, A.; Schlagowski, A.I.; Charles, A.L.; Singh, F.; Bouitbir, J.; Pottecher, J.; Chakfé, N.; Zoll, J.; Geny, B. Mitochondria: Mitochondrial participation in ischemia-reperfusion injury in skeletal muscle. Int. J. Biochem. Cell Biol. 2014, 50, 101-105. [CrossRef] [PubMed]

13. Makris, K.I.; Nella, A.A.; Zhu, Z.; Swanson, S.A.; Casale, G.P.; Gutti, T.L.; Judge, A.R.; Pipinos, I.I. Mitochondriopathy of peripheral arterial disease. Vascular 2007, 15, 336-343. [CrossRef]

14. Bernardi, P. The mitochondrial permeability transition pore: A mystery solved? Front. Physiol. 2013,4 , 95. [CrossRef] [PubMed]

15. Chouchani, E.T.; Pell, V.R.; James, A.M.; Work, L.M.; Saeb-Parsy, K.; Frezza, C.; Krieg, T.; Murphy, M.P. A Unifying Mechanism for Mitochondrial Superoxide Production during Ischemia-Reperfusion Injury. Cell Metab. 2016, 23, 254-263. [CrossRef]

16. Chouchani, E.T.; Pell, V.R.; Gaude, E.; Aksentijević, D.; Sundier, S.Y.; Robb, E.L.; Logan, A.; Nadtochiy, S.M.; Ord, E.N.J.; Smith, A.C.; et al. Ischaemic accumulation of succinate controls reperfusion injury through mitochondrial ROS. Nature 2014, 515, 431-435. [CrossRef]

17. Chouchani, E.T.; Methner, C.; Nadtochiy, S.M.; Logan, A.; Pell, V.R.; Ding, S.; James, A.M.; Cochemé, H.M.; Reinhold, J.; Lilley, K.S.; et al. Cardioprotection by S-nitrosation of a cysteine switch on mitochondrial complex I. Nat. Med. 2013, 19, 753-759. [CrossRef]

18. Pottecher, J.; Kindo, M.; Chamaraux-Tran, T.N.; Charles, A.L.; Lejay, A.; Kemmel, V.; Vogel, T.; Chakfe, N.; Zoll, J.; Diemunsch, P.; et al. Skeletal muscle ischemia-reperfusion injury and cyclosporine A in the aging rat. Fundam. Clin. Pharmacol. 2016, 30, 216-225. [CrossRef]

19. Thaveau, F.; Zoll, J.; Bouitbir, J.; N'guessan, B.; Plobner, P.; Chakfe, N.; Kretz, J.G.; Richard, R.; Piquard, F.; Geny, B. Effect of chronic pre-treatment with angiotensin converting enzyme inhibition on skeletal muscle mitochondrial recovery after ischemia/reperfusion. Fundam. Clin. Pharmacol. 2010, 24, 333-340. [CrossRef]

20. Brandão, M.L.; Roselino, J.E.S.; Piccinato, C.E.; Cherri, J. Mitochondrial alterations in skeletal muscle submitted to total ischemia. J. Surg. Res. 2003, 110, 235-240. [CrossRef]

21. Mansour, Z.; Bouitbir, J.; Charles, A.L.; Talha, S.; Kindo, M.; Pottecher, J.; Zoll, J.; Geny, B. Remote and local ischemic preconditioning equivalently protects rat skeletal muscle mitochondrial function during experimental aortic cross-clamping. J. Vasc. Surg. 2012, 55, 497-505. [CrossRef] [PubMed]

22. Pipinos, I.I.; Swanson, S.A.; Zhu, Z.; Nella, A.A.; Weiss, D.J.; Gutti, T.L.; McComb, R.D.; Baxter, B.T.; Lynch, T.G.; Casale, G.P. Chronically ischemic mouse skeletal muscle exhibits myopathy in association with mitochondrial dysfunction and oxidative damage. Am. J. Physiol. Regul. Integr. Comp. Physiol. 2008, 295, R290-R296. [CrossRef] [PubMed]

23. Schmidt, C.A.; Ryan, T.E.; Lin, C.T.; Inigo, M.M.R.; Green, T.D.; Brault, J.J.; Spangenburg, E.E.; McClung, J.M. Diminished force production and mitochondrial respiratory deficits are strain-dependent myopathies of subacute limb ischemia. J. Vasc. Surg. 2017, 65, 1504-1514.e11. [CrossRef] [PubMed] 
24. Charles, A.L.; Guilbert, A.S.; Guillot, M.; Talha, S.; Lejay, A.; Meyer, A.; Kindo, M.; Wolff, V.; Bouitbir, J.; Zoll, J.; et al. Muscles Susceptibility to Ischemia-Reperfusion Injuries Depends on Fiber Type Specific Antioxidant Level. Front. Physiol. 2017, 8, 52. [CrossRef]

25. Paradis, S.; Charles, A.L.; Georg, I.; Goupilleau, F.; Meyer, A.; Kindo, M.; Laverny, G.; Metzger, D.; Geny, B. Aging Exacerbates Ischemia-Reperfusion-Induced Mitochondrial Respiration Impairment in Skeletal Muscle. Antioxidants (Basel) 2019, 8, 168. [CrossRef]

26. Pottecher, J.; Adamopoulos, C.; Lejay, A.; Bouitbir, J.; Charles, A.L.; Meyer, A.; Singer, M.; Wolff, V.; Diemunsch, P.; Laverny, G.; et al. Diabetes Worsens Skeletal Muscle Mitochondrial Function, Oxidative Stress, and Apoptosis After Lower-Limb Ischemia-Reperfusion: Implication of the RISK and SAFE Pathways? Front. Physiol. 2018, 9, 579. [CrossRef]

27. Koutakis, P.; Miserlis, D.; Myers, S.A.; Kim, J.K.S.; Zhu, Z.; Papoutsi, E.; Swanson, S.A.; Haynatzki, G.; Ha, D.M.; Carpenter, L.A.; et al. Abnormal accumulation of desmin in gastrocnemius myofibers of patients with peripheral artery disease: Associations with altered myofiber morphology and density, mitochondrial dysfunction and impaired limb function. J. Histochem. Cytochem. 2015, 63, 256-269. [CrossRef]

28. Pipinos, I.I.; Judge, A.R.; Zhu, Z.; Selsby, J.T.; Swanson, S.A.; Johanning, J.M.; Baxter, B.T.; Lynch, T.G.; Dodd, S.L. Mitochondrial defects and oxidative damage in patients with peripheral arterial disease. Free Radic. Biol. Med. 2006, 41, 262-269. [CrossRef]

29. Pipinos, I.I.; Sharov, V.G.; Shepard, A.D.; Anagnostopoulos, P.V.; Katsamouris, A.; Todor, A.; Filis, K.A.; Sabbah, H.N. Abnormal mitochondrial respiration in skeletal muscle in patients with peripheral arterial disease. J. Vasc. Surg. 2003, 38, 827-832. [CrossRef]

30. Brass, E.P.; Hiatt, W.R.; Gardner, A.W.; Hoppel, C.L. Decreased NADH dehydrogenase and ubiquinol-cytochrome c oxidoreductase in peripheral arterial disease. Am. J. Physiol. Heart Circ. Physiol. 2001, 280, H603-H609. [CrossRef]

31. Hart, C.R.; Layec, G.; Trinity, J.D.; Le Fur, Y.; Gifford, J.R.; Clifton, H.L.; Richardson, R.S. Oxygen availability and skeletal muscle oxidative capacity in patients with peripheral artery disease: Implications from in vivo and in vitro assessments. Am. J. Physiol. Heart Circ. Physiol. 2018, 315, H897-H909. [CrossRef] [PubMed]

32. Hart, C.R.; Layec, G.; Trinity, J.D.; Kwon, O.S.; Zhao, J.; Reese, V.R.; Gifford, J.R.; Richardson, R.S. Increased skeletal muscle mitochondrial free radical production in peripheral arterial disease despite preserved mitochondrial respiratory capacity. Exp. Physiol. 2018, 103, 838-850. [CrossRef]

33. Morisaki, K.; Furuyama, T.; Matsubara, Y.; Inoue, K.; Kurose, S.; Yoshino, S.; Nakayama, K.; Yamashita, S.; Yoshiya, K.; Yoshiga, R.; et al. External validation of CLI Frailty Index and assessment of predictive value of modified CLI Frailty Index for patients with critical limb ischemia undergoing infrainguinal revascularization. Vascular 2019, 1708538119836005. [CrossRef] [PubMed]

34. Taniguchi, R.; Deguchi, J.; Hashimoto, T.; Sato, O. Sarcopenia as a Possible Negative Predictor of Limb Salvage in Patients with Chronic Limb-Threatening Ischemia. Ann. Vasc. Dis. 2019, 12, 194-199. [CrossRef] [PubMed]

35. Matsubara, Y.; Matsumoto, T.; Aoyagi, Y.; Tanaka, S.; Okadome, J.; Morisaki, K.; Shirabe, K.; Maehara, Y. Sarcopenia is a prognostic factor for overall survival in patients with critical limb ischemia. J. Vasc. Surg. 2015, 61, 945-950. [CrossRef] [PubMed]

36. AlGhatrif, M.; Zane, A.; Oberdier, M.; Canepa, M.; Studenski, S.; Simonsick, E.; Spencer, R.G.; Fishbein, K.; Reiter, D.; Lakatta, E.G.; et al. Lower Mitochondrial Energy Production of the Thigh Muscles in Patients with Low-Normal Ankle-Brachial Index. J. Am. Heart Assoc 2017, 6. [CrossRef] [PubMed]

37. Pipinos, I.I.; Shepard, A.D.; Anagnostopoulos, P.V.; Katsamouris, A.; Boska, M.D. Phosphorus 31 nuclear magnetic resonance spectroscopy suggests a mitochondrial defect in claudicating skeletal muscle. J. Vasc. Surg. 2000, 31, 944-952. [CrossRef] [PubMed]

38. Hou, X.Y.; Green, S.; Askew, C.D.; Barker, G.; Green, A.; Walker, P.J. Skeletal muscle mitochondrial ATP production rate and walking performance in peripheral arterial disease. Clin. Physiol. Funct. Imaging 2002, 22, 226-232. [CrossRef]

39. Lindegaard, B.P.; Bækgaard, N.; Quistorff, B. Mitochondrial dysfunction in calf muscles of patients with combined peripheral arterial disease and diabetes type 2. Int. Angiol. 2017, 36, 482-495.

40. Tecilazich, F.; Dinh, T.; Lyons, T.E.; Guest, J.; Villafuerte, R.A.; Sampanis, C.; Gnardellis, C.; Zuo, C.S.; Veves, A. Postexercise phosphocreatine recovery, an index of mitochondrial oxidative phosphorylation, is reduced in diabetic patients with lower extremity complications. J. Vasc. Surg. 2013, 57, 997-1005. [CrossRef] 
41. Zorov, D.B.; Juhaszova, M.; Sollott, S.J. Mitochondrial ROS-induced ROS release: An update and review. Biochim. Biophys. Acta. 2006, 1757, 509-517. [CrossRef] [PubMed]

42. Lejay, A.; Choquet, P.; Thaveau, F.; Singh, F.; Schlagowski, A.; Charles, A.L.; Laverny, G.; Metzger, D.; Zoll, J.; Chakfe, N.; et al. A new murine model of sustainable and durable chronic critical limb ischemia fairly mimicking human pathology. Eur. J. Vasc. Endovasc. Surg. 2015, 49, 205-212. [CrossRef] [PubMed]

43. Kwon, B.; Kang, C.; Kim, J.; Yoo, D.; Cho, B.R.; Kang, P.M.; Lee, D. $\mathrm{H}_{2} \mathrm{O}_{2}$-responsive antioxidant polymeric nanoparticles as therapeutic agents for peripheral arterial disease. Int. J. Pharm. 2016, 511, 1022-1032. [CrossRef] [PubMed]

44. Lejay, A.; Charles, A.L.; Georg, I.; Goupilleau, F.; Delay, C.; Talha, S.; Thaveau, F.; Chakfe, N.; Geny, B. Critical limb ischemia exacerbates mitochondrial dysfunction in ApoE-/- mice compared to ApoE+/+ mice, but $\mathrm{N}$-acetyl cysteine still confers protection. Eur. J. Vasc. Endovasc. Surg. 2019, 58, 576-582. [CrossRef]

45. Tran, T.P.; Tu, H.; Pipinos, I.I.; Muelleman, R.L.; Albadawi, H.; Li, Y.L. Tourniquet-induced acute ischemia-reperfusion injury in mouse skeletal muscles: Involvement of superoxide. Eur. J. Pharmacol. 2011, 650, 328-334. [CrossRef]

46. Miura, S.; Saitoh, S.; Kokubun, T.; Owada, T.; Yamauchi, H.; Machii, H.; Takeishi, Y. Mitochondrial-Targeted Antioxidant Maintains Blood Flow, Mitochondrial Function, and Redox Balance in Old Mice Following Prolonged Limb Ischemia. Int. J. Mol. Sci. 2017, 18, 1897. [CrossRef]

47. Lejay, A.; Laverny, G.; Paradis, S.; Schlagowski, A.I.; Charles, A.L.; Singh, F.; Zoll, J.; Thaveau, F.; Lonsdorfer, E.; Dufour, S.; et al. Moderate Exercise Allows for shorter Recovery Time in Critical Limb Ischemia. Front. Physiol. 2017, 8, 523. [CrossRef]

48. Guillot, M.; Charles, A.L.; Chamaraux-Tran, T.N.; Bouitbir, J.; Meyer, A.; Zoll, J.; Schneider, F.; Geny, B. Oxidative stress precedes skeletal muscle mitochondrial dysfunction during experimental aortic cross-clamping but is not associated with early lung, heart, brain, liver, or kidney mitochondrial impairment. J. Vasc. Surg. 2014, 60, 1043-1051.e5. [CrossRef]

49. McDermott, M.M.; Peterson, C.A.; Sufit, R.; Ferrucci, L.; Guralnik, J.M.; Kibbe, M.R.; Polonsky, T.S.; Tian, L.; Criqui, M.H.; Zhao, L.; et al. Peripheral artery disease, calf skeletal muscle mitochondrial DNA copy number, and functional performance. Vasc. Med. 2018, 23, 340-348. [CrossRef]

50. Koutakis, P.; Weiss, D.J.; Miserlis, D.; Shostrom, V.K.; Papoutsi, E.; Ha, D.M.; Carpenter, L.A.; McComb, R.D.; Casale, G.P.; Pipinos, I.I. Oxidative damage in the gastrocnemius of patients with peripheral artery disease is myofiber type selective. Redox. Biol. 2014, 2, 921-928. [CrossRef]

51. Weiss, D.J.; Casale, G.P.; Koutakis, P.; Nella, A.A.; Swanson, S.A.; Zhu, Z.; Miserlis, D.; Johanning, J.M.; Pipinos, I.I. Oxidative damage and myofiber degeneration in the gastrocnemius of patients with peripheral arterial disease. J. Transl. Med. 2013, 11, 230. [CrossRef] [PubMed]

52. Brass, E.P.; Wang, H.; Hiatt, W.R. Multiple skeletal muscle mitochondrial DNA deletions in patients with unilateral peripheral arterial disease. Vasc. Med. 2000, 5, 225-230. [CrossRef] [PubMed]

53. Bhat, H.K.; Hiatt, W.R.; Hoppel, C.L.; Brass, E.P. Skeletal muscle mitochondrial DNA injury in patients with unilateral peripheral arterial disease. Circulation 1999, 99, 807-812. [CrossRef] [PubMed]

54. Hsu, S.L.; Yin, T.C.; Shao, P.L.; Chen, K.H.; Wu, R.W.; Chen, C.C.; Lin, P.Y.; Chung, S.Y.; Sheu, J.J.; Sung, P.H.; et al. Hyperbaric oxygen facilitates the effect of endothelial progenitor cell therapy on improving outcome of rat critical limb ischemia. Am. J. Transl. Res. 2019, 11, 1948-1964.

55. Sheu, J.J.; Lee, F.Y.; Wallace, C.G.; Tsai, T.H.; Leu, S.; Chen, Y.L.; Chai, H.T.; Lu, H.I.; Sun, C.K.; Yip, H.K. Administered circulating microparticles derived from lung cancer patients markedly improved angiogenesis, blood flow and ischemic recovery in rat critical limb ischemia. J. Transl. Med. 2015, 13. [CrossRef]

56. Tetsi, L.; Charles, A.L.; Georg, I.; Goupilleau, F.; Lejay, A.; Talha, S.; Maumy-Bertrand, M.; Lugnier, C.; Geny, B. Effect of the Phosphodiesterase 5 Inhibitor Sildenafil on Ischemia-Reperfusion-Induced Muscle Mitochondrial Dysfunction and Oxidative Stress. Antioxidants (Basel) 2019, 8, 93. [CrossRef]

57. Lejay, A.; Paradis, S.; Lambert, A.; Charles, A.L.; Talha, S.; Enache, I.; Thaveau, F.; Chakfe, N.; Geny, B. N-Acetyl Cysteine Restores Limb Function, Improves Mitochondrial Respiration, and Reduces Oxidative Stress in a Murine Model of Critical Limb Ischaemia. Eur. J. Vasc. Endovasc. Surg. 2018, 56, 730-738. [CrossRef]

58. Masud, R.; Shameer, K.; Dhar, A.; Ding, K.; Kullo, I.J. Gene expression profiling of peripheral blood mononuclear cells in the setting of peripheral arterial disease. J. Clin. Bioinforma. 2012, 2, 6. [CrossRef] 
59. Mitchell, R.G.; Duscha, B.D.; Robbins, J.L.; Redfern, S.I.; Chung, J.; Bensimhon, D.R.; Kraus, W.E.; Hiatt, W.R.; Regensteiner, J.G.; Annex, B.H. Increased levels of apoptosis in gastrocnemius skeletal muscle in patients with peripheral arterial disease. Vasc. Med. 2007, 12, 285-290. [CrossRef]

60. Gardner, A.W.; Parker, D.E.; Montgomery, P.S.; Sosnowska, D.; Casanegra, A.I.; Ungvari, Z.; Csiszar, A.; Sonntag, W.E. Greater Endothelial Apoptosis and Oxidative Stress in Patients with Peripheral Artery Disease. Int. J. Vasc. Med. 2014, 2014. [CrossRef]

61. Skórkowska-Telichowska, K.; Adamiec, R.; Tuchendler, D.; Gasiorowski, K. Susceptibility to apoptosis of lymphocytes from patients with peripheral arterial disease. Clin. Investig. Med. 2009, 32, E345-E351. [CrossRef] [PubMed]

62. Gardner, A.W.; Parker, D.E.; Montgomery, P.S.; Sosnowska, D.; Casanegra, A.I.; Esponda, O.L.; Ungvari, Z.; Csiszar, A.; Sonntag, W.E. Impaired vascular endothelial growth factor A and inflammation in patients with peripheral artery disease. Angiology 2014, 65, 683-690. [CrossRef] [PubMed]

63. Alves-Cabratosa, L.; Garcia-Gil, M.; Comas-Cufí, M.; Blanch, J.; Ponjoan, A.; Martí-Lluch, R.; Elosua-Bayes, M.; Parramon, D.; Camós, L.; Ramos, R. Role of Low Ankle-Brachial Index in Cardiovascular and Mortality Risk Compared with Major Risk Conditions. J. Clin. Med. 2019, 8, 870. [CrossRef] [PubMed]

(C) 2019 by the authors. Licensee MDPI, Basel, Switzerland. This article is an open access article distributed under the terms and conditions of the Creative Commons Attribution (CC BY) license (http://creativecommons.org/licenses/by/4.0/). 